ESAIM: PROCEEDINGS, May 2009, Vol. 27, p. 138-155

C. Besse, O. Goubet, T. Goudon \& S. Nicaise, Editors

\title{
CALCUL DE LA SENSIBILITÉ D'ORDRE DEUX D'UNE OBSERVATION AÉRODYNAMIQUE
}

\author{
Ludovic Martin $^{1,2}$ AND Gilbert Roge ${ }^{1}$
}

\begin{abstract}
This paper outlines a new formulation for Hessian calculation for a functional of interest for aerodynamic applications with regards to shape parameters. An analysis of the order of the computational cost and memory required shows that an industrial use is possible. After having described the implementation strategy, we validate our code with two test cases (fuselage and ONERA M6 wing).

Résumé. Cet article présente une nouvelle formulation permettant de calculer la matrice hessienne d'une observation aérodynamique par rapport à des paramètres de définition de la géométrie. Une analyse du nombre d'opérations à réaliser ainsi que de l'espace mémoire nécessaire montre que son utilisation industrielle s'avère possible. Après avoir présenté la stratégie d'implémentation, nous validons cette sensibilité seconde sur deux cas-test (fuselage et aile ONERA M6).
\end{abstract}

\section{INTRODUCTION}

L'utilisation des sensibilités entraîne des améliorations considérables dans divers domaines. Il y a bien sûr le champ de l'optimisation de forme [3] et [4] avec comme critère de qualité la valeur d'une ou plusieurs quantitées aérodynamiques d'intérêt (coefficient de portance le plus fort possible ou de traînée le plus faible possible par exemple). Un autre domaine d'application est la constitution d'un modèle approché. Pour cela, plusieurs méthodes existent, se répartissant en deux classes principales: les méthodes globales [5] [6] (RBF (Radial Basis Function), Kriging, ...) et locales (développement de Taylor ou de Padé, ...). Il s'agit de constituer, à partir d'un certain nombre de calculs exacts, une fonction d'approximation donnant la valeur d'une observation aérodynamique sur une certaine plage de variation des paramètres d'entrée. Les sensibilités permettent de réduire le nombre de données utilisées, et donc de diminuer encore le coût de la méthode sans dégrader l'approximation. Enfin, nous considérons la propagation des incertitudes. Il s'agit de voir comment réagit l'observation de notre choix à une incertitude sur un ou plusieurs paramètres d'entrée. Pour cela, on se donne une distribution de probabilité pour chaque paramètre incertain et on applique soit les méthodes de type Monte-Carlo approché (en utilisant un modèle approché), soit la méthode des moments (calcul direct de la moyenne et de l'écart-type: F(S)OSM: First (Second) Order Second Moment) ou encore des méthodes adaptées au calcul de queues de probabilité (F(S)ORM: First (Second) Order Reliability Method). Les dérivées rendent chacune de ces techniques beaucoup plus efficaces (elles sont même à la base de la méthode des moments, qui s'appuie sur un développement de Taylor).

Ayant en tête l'intérêt du calcul des sensibilités, on peut légitimement se demander jusqu'à quel ordre il est pertinent d'aller. Plus précisement, l'ordre deux a-t-il une réelle utilité par rapport à l'ordre un ? Prenons deux exemples motivant l'ordre deux. Le premier concerne l'optimisation. On suppose pour simplifier que notre

1 Dassault Aviation, 78 quai Marcel Dassault, 92552 Saint-Cloud, FRANCE

2 Université Paul Sabatier, 118 route de Narbonne, 31062 Toulouse, FRANCE

(C) EDP Sciences, SMAI 2009 
résultat ne se situe pas sur un bord du domaine admissible. Pour éviter de prendre un point d'inflexion pour un optimum local, la nullité de la dérivée première de la fonction d'optimisation ne suffit pas. Il faut utiliser les conditions d'optimalité d'ordre 2. Le calcul de la dérivée seconde va également permettre d'appliquer la méthode de Newton (au lieu d'une méthode de type quasi-Newton). Le second exemple motivant le calcul de la dérivée seconde concerne les études d'incertitude. Il est essentiel de connaître précisement autour de quelle valeur moyenne notre fonction va fluctuer. Généralement, on considère une distribution centrée en entrée. Ainsi, en se limitant à l'ordre un, la méthode des moments ne prévoit aucun impact sur la moyenne de notre fonction d'intérêt qui reste donc égale à la valeur sans incertitude. La perturbation de la moyenne est en effet au minimum d'ordre deux.

Ainsi, la détermination de la sensibilité seconde apporte une vraie valeur ajoutée dans bien des domaines. Sa détermination demande de différentier l'ensemble de la chaîne permettant de passer d'un jeu de paramètres aérodynamiques (n'influant pas sur le maillage) ou géométriques (influant sur le maillage) aux observables à différentier. La figure 1 récapitule les principales étapes, différentes suivant le type de paramètre considéré. Cependant, pour être utilisable industriellement, le calcul doit être optimal tant sur le plan de la complexité

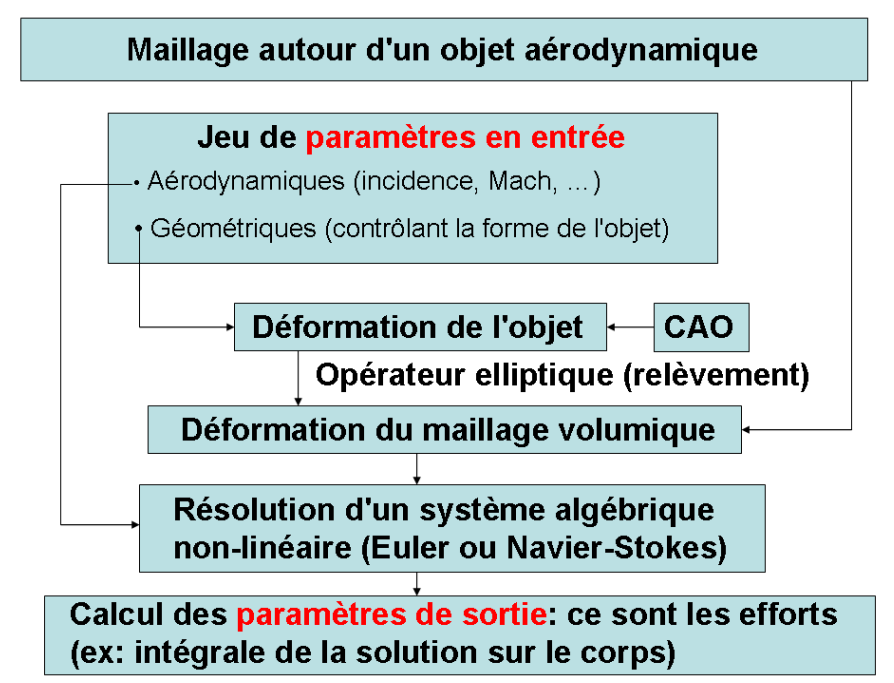

Figure 1. Chaîne à différentier

(nombre d'opérations à effectuer) que sur l'espace mémoire nécessaire. On veillera en particulier à ne jamais avoir à manipuler des objets de taille égale au nombre de sommets du maillage élevé à une puissance strictement supérieure à un et ainsi ne pas compromettre la viabilité industrielle de l'ordre deux.

On va décrire dans cet article la formulation de la dérivée seconde pour deux types différents de paramètres. On s'inspirera pour cela de la technique de Giles [1] en l'étendant au cas où deux adjoints sont utilisés (un adjoint Euler (ou Navier-Stokes) et un adjoint de l'opérateur elliptique servant pour la déformation de maillage volumique). La stratégie d'implémentation sera ensuite évoquée. Enfin, nous présenterons les résultats numériques obtenus pour nos deux cas de validation. Notons que les fonctions manipulées sont toutes supposées au moins deux fois dérivables.

\section{ECRITURE DE LE DÉRIVÉE SECONDE}

\subsection{Position du problème}

Commençons par poser les notations utilisées tout au long de cet article. Le système à résoudre correspond aux équations d'Euler (ou de Navier Stokes) modélisant l'écoulement d'un fluide compressible en régime stationnaire. 
On se place dans l'espace $\mathbb{R}^{n}$, l'entier $n$ désignant sa dimension et pouvant être égal à 2 ou 3 . Le maillage utilisé comporte $N_{M}$ sommets.

Le problème à résoudre comporte $2+n$ équations (1 pour la densité, $\mathrm{n}$ autres pour la quantitée de mouvement et une dernière pour l'énergie). Ce système d'équations, noté $E$, s'écrit:

$$
E(\eta, \nu, w)=0
$$

où $w$ désigne l'état du système fluide et $\eta$ (resp. $\nu$ ) désigne la classe des paramètres d'entrée qui ont une influence nulle (resp. non nulle) sur le maillage. Par exemple, le nombre de Mach ou les grandeurs décrivant la nature de l'écoulement sont à ranger dans les variables $\eta$ tandis que les paramètres contrôlant la forme de l'objet appartiennent à l'autre classe $\nu$. Nous désignerons par $N_{\eta}$ (resp. par $N_{\nu}$ ) le nombre de paramètres de type $\eta$ (resp. de type $\nu$ ).

Plus précisement, on va supposer que la classe de paramètres $\nu$ n'intervient dans le système d'équations $E$ qu'au travers des coordonnées du maillage sous forme d'un champ de déplacement volumique noté $D$. Ainsi, en écrivant toutes les dépendances (explicites et implicites), on arrive à l'écriture suivante:

$$
E(\eta, D(\nu), w(\eta, D(\nu)))=0
$$

Nous distinguerons ici deux cas concernant le calcul du champ $D$ de déplacement volumique. Le premier s'applique lorsque $D$ est calculé directement (comme dans le cas d'une rotation globale de maillage). Le second, en revanche, demande de passer par le calcul d'un champ $d$ de déplacement surfacique définit sur l'objet autour duquel l'écoulement est simulé. Ce calcul est assuré par le modeleur géométrique qui, en plus de paramétrer la géométrie du corps, fait suivre (par déformation, en utilisant un opérateur de type Laplace-Beltrami) le maillage surfacique. C'est le processus voué à être le plus utilisé en pratique. Dans ce cas, on introduit un nouvel opérateur $L$ chargé d'étendre le déplacement surfacique au maillage tout entier. Ce problème s'écrit:

$$
L(D, d)=0
$$

Généralement, c'est un opérateur elliptique du second ordre (comme l'opérateur d'élasticité linéaire) qui joue le rôle de $L$.

Enfin, les fonctions d'intérêt, au nombre de $N_{g}$, seront notées $g$. De même que pour $E$, on a les dépendances suivantes:

En résumé, on a en entrée:

$$
g(\eta, \nu)=G(\eta, D(\nu), w(\eta, D(\nu)))
$$

$$
\left[\left(\eta_{i}\right)_{1 \leq i \leq N_{\eta}},\left(\nu_{i}\right)_{1 \leq i \leq N_{\nu}}\right]
$$

et en sortie:

$$
\left(g_{i}\right)_{1 \leq i \leq N_{g}}
$$

Il est utile de donner ici pour un cas réel ${ }^{1}$ les ordres de grandeur concernant les entiers manipulés:

$$
\left\{\begin{array}{l}
N_{M} \sim 10^{6} \\
N_{\eta} \sim 10 \\
N_{\nu} \sim 100 \\
N_{g} \sim 10
\end{array}\right.
$$

C'est donc le nombre $N_{M}$ de sommets qui s'avère être le nombre prépondérant. L'objectif est la détermination des sensibilités première et seconde des observables $g$ par rapport aux deux classes de paramètres $\eta$ et $\nu$, sachant que le nombre d'opérations à effectuer devra rester compatible d'une utilisation industrielle.

\footnotetext{
${ }^{1}$ Dans les exemples traités, on se contentera de prendre 2 paramètres et 1 observation.
} 


\subsection{Sensibilités première et seconde pour des paramètres n'influençant pas le maillage}

Il s'agit donc de trouver les expressions des dérivées première et seconde de l'observable $g_{k}(\eta)=G_{k}(\eta, w(\eta))$ ( $k$ variant de 1 a $N_{g}$ ) par rapport aux paramètres de type $\eta$. L'équation d'état considéré s'écrit:

$$
E(\eta, w(\eta))=0
$$

La différentiation par rapport à $\eta_{i}\left(i\right.$ variant de 1 à $\left.N_{\eta}\right)$ de cette équation d'état donne:

$$
\frac{\partial E}{\partial \eta_{i}}+\frac{\partial E}{\partial w} \frac{\partial w}{\partial \eta_{i}}=0
$$

On en déduit l'expression suivante de la sensibilité d'ordre un vis-à-vis de $\eta_{i}$ :

$$
\frac{\partial g_{k}}{\partial \eta_{i}}=\frac{\partial G_{k}}{\partial \eta_{i}}-\psi_{k}^{T} \frac{\partial E}{\partial \eta_{i}}
$$

où $\psi_{k}$ désigne l'adjoint "Euler" (ou "Navier-Stokes") de la $k^{\text {ème }}$ observation, solution de l'équation linéaire suivante:

$$
\left(\frac{\partial E}{\partial w}\right)^{T} \psi_{k}=\left(\frac{\partial G_{k}}{\partial w}\right)^{T}
$$

En différentiant par rapport à $\eta_{j}\left(j\right.$ variant de 1 à $\left.N_{\eta}\right)$ l'expression (7) concernant l'équation d'état $E$, il vient:

$$
\frac{\partial E}{\partial w} \frac{\partial^{2} w}{\partial \eta_{i} \partial \eta_{j}}+K_{\eta_{i} \eta_{j}}^{2} E=0
$$

où $K_{\eta_{i} \eta_{j}}^{2}$ désigne l'opérateur de dérivées partielles secondes suivant:

$$
K_{a b}^{2} f=\frac{\partial^{2} f}{\partial a \partial b}+\frac{\partial^{2} f}{\partial a \partial w} \frac{\partial w}{\partial b}+\frac{\partial^{2} f}{\partial w \partial b} \frac{\partial w}{\partial a}+\frac{\partial^{2} f}{\partial w \partial w}\left(\frac{\partial w}{\partial a} ; \frac{\partial w}{\partial b}\right)
$$

Dans cette expression, la notation suivante est utilisée:

$$
A(x ; y)=x^{T} A y
$$

Nanti de cette information, on peut exprimer la sensibilité seconde vis-à-vis de $\eta_{i}$ et $\eta_{j}$ (cf Giles):

$$
\frac{\partial^{2} g_{k}}{\partial \eta_{i} \eta_{j}}=K_{\eta_{i} \eta_{j}}^{2} G_{k}-\psi_{k}^{T} K_{\eta_{i} \eta_{j}}^{2} E
$$

Faisons le bilan du nombre d'opérations nécessaires à la détermination de ces deux dérivées. Les systèmes évoqués sont de taille proportionnelle au nombre $N_{M}$ de sommets du maillage.

- Une solution de système algébrique non-linéaire pour $w$, système ayant $(2+n) N_{M}$ équations à $(2+n) N_{M}$ inconnues

- $N_{\eta}$ solutions de systèmes linéaires, chacun de dimension $(2+n) N_{M}$, pour $\frac{\partial w}{\partial \eta_{i}}$

- $N_{g}$ solutions de systèmes adjoints, chacun de dimension $(2+n) N_{M}$, pour $\psi_{k}$

- $N_{g} N_{\eta}^{2}\left(\right.$ resp. $\left.N_{\eta}^{2}\right)$ évaluations pour $K_{\eta_{i} \eta_{j}}^{2} G_{k}\left(\right.$ resp. $\left.K_{\eta_{i} \eta_{j}}^{2} E\right)$

- $N_{g} N_{\eta}^{2}$ produits scalaires pour $\psi_{k}^{T} K_{\eta_{i} \eta_{j}}^{2} E$

Le coût d'une évaluation ou d'un produit scalaire étant négligeable devant la résolution d'un système, on arrive à un nombre de systèmes linéaires (de dimension $(2+n) N_{M}$ ) égal à $N_{g}+N_{\eta}$ (sans compter la résolution du système non-linéaire), ce qui est viable industriellement. 


\subsection{Sensibilités première et seconde pour des paramètres influençant le maillage}

Cette section est le coeur même de cet article. Il s'agit de la détermination des dérivées première et seconde de l'observable $g_{k}\left(k\right.$ variant de 1 à $N_{g}$ ) par rapport aux paramètres de type $\nu$. Ici, deux cas de figures peuvent apparaître:

- Cas 1: le champ de déplacement volumique $D$ est obtenu directement

- Cas 2: le champ de déplacement volumique $D$ est obtenu via un champ de déplacement surfacique $d$ issu d'un modeleur géométrique (CAO de l'objet). Ce modeleur paramètre la forme de l'objet et fait suivre le maillage surfacique par déformation. On utilise généralement un opérateur de type Laplace-Beltrami.

Les données de départ sont donc:

$$
\left\{\begin{array}{l}
g(\nu)=G(D(\nu), w(D(\nu))) \\
E(D(\nu), w(D(\nu)))=0 \\
L(D(\nu), d(\nu))=0(\operatorname{cas} 2)
\end{array}\right.
$$

La différentiation de l'équation d'état $E$ par rapport à $\nu_{i}\left(i\right.$ variant de 1 à $\left.N_{\nu}\right)$ donne:

$$
\frac{\partial E}{\partial D} \frac{\partial D}{\partial \nu_{i}}+\frac{\partial E}{\partial w} \frac{\partial w}{\partial \nu_{i}}=0
$$

Pour le second cas envisagé, on a également besoin de différentier par rapport à $\nu_{i}$ l'opérateur de déformation $L$ :

$$
\frac{\partial L}{\partial D} \frac{\partial D}{\partial \nu_{i}}+\frac{\partial L}{\partial d} \frac{\partial d}{\partial \nu_{i}}=0
$$

On en déduit les expressions suivantes de la sensibilité d'ordre un de $g$ vis-à-vis de $\nu_{i}$ :

$$
\left\{\begin{array}{l}
\frac{\partial g_{k}}{\partial \nu_{i}}=\left[\frac{\partial G_{k}}{\partial D}-\psi_{k}^{T} \frac{\partial E}{\partial D}\right] \frac{\partial D}{\partial \nu_{i}}(\operatorname{cas} 1) \\
\frac{\partial g_{k}}{\partial \nu_{i}}=-\phi_{k}^{T} \frac{\partial L}{\partial d} \frac{\partial d}{\partial \nu_{i}}(\operatorname{cas} 2)
\end{array}\right.
$$

où $\phi_{k}$ désigne l'adjoint "déformation" de la $k^{\text {ème }}$ observation, solution de l'équation linéaire suivante:

$$
\left(\frac{\partial L}{\partial D}\right)^{T} \phi_{k}=\left(\frac{\partial G_{k}}{\partial D}\right)^{T}-\left(\frac{\partial E}{\partial D}\right)^{T} \psi_{k}
$$

Différentier par rapport à $\nu_{j}\left(j\right.$ variant de 1 à $\left.N_{\nu}\right)$ l'expression (14) concernant l'équation d'état $E$ permet d'aboutir à l'expression suivante:

$$
F_{w \nu_{i} \nu_{j}}^{2} E+\frac{\partial E}{\partial D} \frac{\partial^{2} D}{\partial \nu_{i} \partial \nu_{j}}+\frac{\partial E}{\partial w} \frac{\partial^{2} w}{\partial \nu_{i} \partial \nu_{j}}=0
$$

où $F_{w \nu_{i} \nu_{j}}^{2}$ désigne l'opérateur de dérivées partielles secondes suivant:

$$
F_{c a b}^{2} f=\frac{\partial^{2} f}{\partial D^{2}}\left(\frac{\partial D}{\partial a} ; \frac{\partial D}{\partial b}\right)+\frac{\partial^{2} f}{\partial D \partial c}\left(\frac{\partial c}{\partial a} ; \frac{\partial D}{\partial b}\right)+\frac{\partial^{2} f}{\partial c \partial D}\left(\frac{\partial D}{\partial a} ; \frac{\partial c}{\partial b}\right)+\frac{\partial^{2} f}{\partial c^{2}}\left(\frac{\partial c}{\partial a} ; \frac{\partial c}{\partial b}\right)
$$

Pour le second cas envisagé, il faut aussi différentier par rapport à $\nu_{j}$ l'expression (15) faisant intervenir l'opérateur de déformation $L$ :

$$
F_{d \nu_{i} \nu_{j}}^{2} L+\frac{\partial L}{\partial D} \frac{\partial^{2} D}{\partial \nu_{i} \partial \nu_{j}}+\frac{\partial L}{\partial d} \frac{\partial^{2} d}{\partial \nu_{i} \partial \nu_{j}}=0
$$


De là, on peut déterminer la sensibilité seconde de l'observation vis-à-vis de $\nu_{i}$ et $\nu_{j}$ :

$$
\left\{\begin{array}{l}
\frac{\partial^{2} g_{k}}{\partial \nu_{i}^{2}}=F_{w \nu_{i} \nu_{j}}^{2} G_{k}-\psi_{k}^{T} F_{w \nu_{i} \nu_{j}}^{2} E+\left[\frac{\partial G_{k}}{\partial D}-\psi_{k}^{T} \frac{\partial E}{\partial D}\right] \frac{\partial^{2} D}{\partial \nu_{i} \nu_{j}}(\operatorname{cas} 1) \\
\frac{\partial^{2} g_{k}}{\partial \nu_{i}^{2}}=F_{w \nu_{i} \nu_{j}}^{2} G_{k}-\psi_{k}^{T} F_{w \nu_{i} \nu_{j}}^{2} E-\phi_{k}^{T}\left[F_{d \nu_{i} \nu_{j}}^{2} L+\frac{\partial L}{\partial d} \frac{\partial^{2} d}{\partial \nu_{i} \nu_{j}}\right](\operatorname{cas} 2)
\end{array}\right.
$$

Faisons donc le bilan du coût total nécessaire à la détermination des sensibilités première et seconde. Les systèmes évoqués sont de taille proportionnelle au nombre $N_{M}$ de sommets du maillage.

- Une solution de système non-linéaire pour $w$, système ayant $(2+n) N_{M}$ équations à $(2+n) N_{M}$ inconnues

- $N_{\nu}$ solutions de systèmes linéaires, chacun de dimension $(2+n) N_{M}$, pour $\frac{\partial w}{\partial \nu_{i}}$

- $N_{g}$ solutions de systèmes adjoints, chacun de dimension $(2+n) N_{M}$, pour $\psi_{k}$

- $N_{g}$ solutions de systèmes adjoints, chacun de dimension $n N_{M}$, pour $\phi_{k}$ (cas 2)

- $N_{g} N_{\nu}^{2}\left(\right.$ resp. $\left.N_{\nu}^{2}\right)$ évaluations pour $F_{w \nu_{i} \nu_{j}}^{2} G_{k}\left(\right.$ resp. $\left.F_{w \nu_{i} \nu_{j}}^{2} E\right)$

- $N_{g} N_{\nu}^{2}$ produits scalaires pour $\psi_{k}^{T} F_{w \nu_{i} \nu_{j}}^{2} E$

- $N_{g}$ évaluations pour $\frac{\partial G_{k}}{\partial D}$ et $\psi_{k}^{T} \frac{\partial E}{\partial D}$ (cas 1$)$

- $N_{\nu}^{2}$ évaluations pour $F_{d \nu_{i} \nu_{j}}^{2} L$ et $\frac{\partial L}{\partial d} \frac{\partial^{2} d}{\partial \nu_{i} \nu_{j}}$ (cas 2)

- $N_{g} N_{\nu}^{2}$ produits scalaires pour $\left[\frac{\partial G_{k}}{\partial D}-\psi_{k}^{T} \frac{\partial E}{\partial D}\right] \frac{\partial^{2} D}{\partial \nu_{i} \nu_{j}}(\operatorname{cas} 1)$

- $N_{g} N_{\nu}^{2}$ produits scalaires pour $\phi_{k}^{T}\left[F_{d \nu_{i} \nu_{j}}^{2} L+\frac{\partial L}{\partial d} \frac{\partial^{2} d}{\partial \nu_{i} \nu_{j}}\right]$ (cas 2)

La taille de chacun des systèmes linéaires à résoudre est proportionelle au nombre de sommets $N_{M}$ du maillage. Le coût d'une évaluation ou d'un produit scalaire étant négligeable devant la résolution d'un système, on arrive à un nombre de systèmes linéaires égal à $N_{g}+N_{\nu}$ dans le premier cas et à $2 N_{g}+N_{\nu}$ dans le second cas (sans compter la résolution du système non-linéaire). Ce nombre de systèmes linéaires à résoudre reste donc très inférieur au nombre de sommets du maillage $N_{M}$, ce qui rend cette formulation utilisable pour des applications industrielles.

Remarquons que, dans l'établissement de nos formulations, nous utilisons $E=0, \delta E=0$ et $\delta^{2} E=0$ (ainsi que $L=0, \delta L=0$ et $\delta^{2} L=0$ ). En fait, dans un cadre discret, l'équation d'état $E=0$ (et de déformation $L=0$ ) est résolue avec une certaine préçision. De plus, nous n'utilisons pas le gradient continu mais discret (différentiation des équations discrètes et non discrétisation du gradient continu). Ainsi, $E, \delta E$ et $\delta^{2} E$ (ainsi que $L, \delta L$ et $\delta^{2} L$ ) ne sont pas rigoureusement nuls. L'utiliser introduit une approximation dans la formulation. Il existe une autre formulation évitant d'utiliser $\delta^{2} E=0\left(\right.$ et $\left.\delta^{2} L=0\right)$. Cette alternative demande néanmoins la résolution de systèmes linéaires supplémentaires (calcul de la dérivée des adjoints $\psi$ et $\phi$ ). Nous nous sommes naturellement tournés vers la formulation la moins coûteuse.

\section{IMPLÉMENTATION DANS UN CODE INDUSTRIEL}

\subsection{Rappel des équations}

La simulation concerne l'écoulement d'un fluide autour d'un corps rigide. On note $\Omega$ l'espace occupé par le fluide simulé. Nous nous intéressons ici aux équations d'Euler tridimensionnelles non linéaires permettant de modéliser l'écoulement des fluides compressibles non visqueux et non conducteurs de chaleur. Dans ce contexte, le système des équations d'Euler $E$ s'écrit (formulation conservative):

$$
\frac{\partial w}{\partial t}+\nabla \cdot F(w)=0 \text { dans } \Omega
$$


On rappelle ci-dessous les expressions du vecteur d'état $w \in \mathbb{R}^{5}$ et de la fonction de flux $F(w) \in\left(\mathbb{R}^{5}\right)^{3}$.

$$
w=\left\{\begin{array}{l}
\rho \\
\rho V \\
E
\end{array}\right\} \quad F(w)=\left\{\begin{array}{l}
\rho V \\
\rho V \otimes V+P I d_{3} \\
(E+P) V
\end{array}\right\}
$$

où $\rho$ est la densité volumique du fluide, $E$ l'énergie totale par unité de volume, $P$ la pression statique et $V$ la vitesse. La notation $I d_{3}$ désigne la matrice identité en dimension 3.

Concernant les conditions aux limites, trois types de frontière sont à considérer:

- La frontière $\Gamma_{c}$ sépare le fluide du corps. Une condition de glissement est ici imposée: $V \cdot n=0$ où $n$ désigne le vecteur normal au corps.

- Afin de limiter les calculs, on construit une frontière artificielle $\Gamma_{\infty}$ appelée "frontière infinie" qui sera située à une distance raisonnable du corps (pas trop près pour ne pas perturber les phénomènes physiques). On considèrera que l'écoulement rentrant, noté $w_{\infty}$, est uniforme. En utilisant l'hyperbolicité des équations d'Euler, cette condition sera imposée de manière faible sur les caractéristiques rentrantes.

- Toujours dans le but de limiter notre domaine de calcul, les éventuelles propriétés de symétrie des corps étudiés seront prises en compte en créant des frontières $\Gamma_{\text {sym }}$ au niveau des plans de symétrie. Des conditions de symétrie y sont imposées.

\subsection{Stratégie de résolution}

La stratégie de résolution décrite ici correspond à celle qui est implémentée dans le code industriel Euler Eugenie, code propriétaire de Dassault Aviation.

Les cas qui nous occupent étant stationnaires, on suppose que le terme $\frac{\partial w}{\partial t}$ est nul. On résoud donc:

$$
\left\{\begin{array}{l}
\nabla \cdot F(w)=0 \text { dans } \Omega \\
+ \text { conditions aux limites }
\end{array}\right.
$$

Pour résoudre ce problème non linéaire, nous nous intéressons à la solution stationnaire du problème pseudoinstationnaire correspondant, qui sera résolu en utilisant un pas de temps local. Le domaine de calcul $\Omega$ est discrétisé par un maillage composé de tétraèdres. L'intégration spatiale utilise la méthode dite de Galerkin volumesfinis. Une décomposition du domaine de calcul en sous-domaines (partition des tétraèdres de la triangulation) est alors réalisée. Typiquement, on découpe le maillage en 32, 64 ou 128 sous-domaines. Le calcul est ensuite mené sur un ordinateur multi-processeurs à mémoire distribuée. Chaque sous-domaine est associé à un processeur. La communication entre les processeurs est assurée par la bibliothèque MPI (Message Passing Interface) et permet d'obtenir la même solution qu'en mono-domaine (calcul séquentiel).

Plusieurs flux numériques (Osher, Jameson, Roe, ...) sont à notre disposition. Notre choix s'est naturellement porté vers un schéma modifié de Lax-Wendroff, flux le plus utilisé chez Dassault Aviation car présentant un bon compromis entre robustesse et précision. Plus précisement, il s'agit d'un schéma prédicteur-correcteur centré d'ordre deux en espace auquel on ajoute un terme de dissipation. Notons que le terme de bord sur $\Gamma_{\infty}$ est calculé en utilisant le flux de Steger-Warming.

Concernant la résolution des systèmes linéaires pour déterminer les solutions directes et adjointes, nous utilisons le solveur itératif GMRes (General Minimum Residual) associé à un préconditionnement de type bloc I.L.D.U. (Incomplete Lower-Diagonal-Upper).

Ainsi, avec le code de calcul Eugenie, nous disposons, pour chaque nouveau jeu de variables d'entrée, de la réponse aérodynamique ainsi que des sensibilités première et seconde de celle-ci par rapport aux paramètres choisis, de type $\eta$ ou $\nu$ (cf section précédente pour la définition). 


\subsection{Etapes de la différentiation}

Le travail consiste à coder dans Eugenie la détermination des sensibilités secondes pour les deux classes $\eta$ et $\nu$ de paramètres. On distingue trois principales étapes dans la construction et la validation de ces grandeurs:

- Etape 1: construction par différentiation automatique des routines

- Etape 2: validation partielle en comparant avec les résultats obtenus par différences finies

- Etape 3: validation globale par l'utilisation de cas-test de référence

Commençons par décrire la méthode utilisée lors de l'étape 1 pour différentier de façon automatique les codes (écrits en FORTRAN). Le point de départ est l'ensemble des routines utilisées pour le calcul de la sensibilité première. On note qu'il n'y a pas de nouvelle résolution de systèmes à mettre en place en passant de l'ordre deux à l'ordre un.

L'outil principal de cette étape est le logiciel de différentiation "automatique" Tapenade, développé par l'INRIA [2], qui opère par transformation du code source. Deux modes de fonctionnement existent, à savoir le mode direct et le mode inverse. Nous avons fait le choix du premier mode cité, plus intuitif, qui marche comme suit. Si on donne une liste de variables d'entrée et un ensemble d'unités de programme écrit en Fortran calculant une fonction numérique $f$, alors Tapenade génère un code Fortran augmenté qui recalcule la fonction et qui donne ses dérivées directionnelles. Ici, la fonction $f$ sera déjà une dérivée directionnelle, puisqu'il s'agit d'appliquer Tapenade à des routines initialement différentiées.

Par exemple, pour calculer la dérivée seconde du résidu Euler $E$ par rapport au champ de déplacement volumique $D$ (ie par rapport aux coordonnées des sommets du maillage), il faut différentier la routine eufluxdx calculant la dérivée première. Cette dernière est elle-même issue de la différentiation de eu flux [8] calculant le flux Euler. Elle se compose des sous-routines suivantes:

- eufllx: calcul du flux de Lax-Wendroff

- eulgl0: intégrale de pression provenant de la condition de glissement sur le corps

- euling: condition aux limites sur $\Gamma_{\infty}$ par le flux de Steger-Warming (demi solveur de Riemann)

Au préalable, il ne faut pas oublier de différentier une seconde fois les routines de construction géométrique, dont les principales sont:

- eucgli: calcul des normales à la surface de l'objet

- eucdsg: calcul des longueurs des barres du maillage volumique

- eucvls: calcul des volumes des cellules du maillage volumique

Une fois différentiée une routine, il faut la valider isolément (étape 2 du processus). Pour cela, nous comparons le résultat obtenu par Tapenade avec une approximation par différences finies. Supposons que la routine en question calcule la fonction $g(x)$ où $x$ désigne les paramètres d'entrée de différentiation. Notons $D^{k} g_{D F}\left(\epsilon_{D F}\right)$ (resp. $D^{k} g_{D A}$ ) la différentielle d'ordre $k$ de $g$ obtenue par la méthode des différences finies avec un pas noté $\epsilon_{D F}$ (resp. par différentiation automatique). La figure 2 montre l'erreur relative $\frac{\left\|D^{k} g_{D F}\left(\epsilon_{D F}\right)-D^{k} g_{D A}\right\|}{\left\|D^{k} g_{D A}\right\|} \operatorname{sur}$ la différentielle d'ordre $k=1$ (à gauche) et sur celle d'ordre $k=2$ (à droite) quand on fait varier le paramètre $\epsilon_{D F}$. Pour la différentielle ordre 1 , on utilise le schéma centré $\frac{g\left(x+\epsilon_{D F}\right)-g(x)}{\epsilon_{D F}}$ (rouge) et décentré $\frac{g\left(x+\epsilon_{D F}\right)-g\left(x-\epsilon_{D F}\right)}{2 \epsilon_{D F}}$ (vert) tandis que pour celle d'ordre 2, on fait appel au schéma centré $\frac{g\left(x+\epsilon_{D F}\right)-2 g(x)+g\left(x-\epsilon_{D F}\right)}{\epsilon_{D F}^{2}}$. La forme des courbes obtenues (qualifiées de "courbe en V") montre une décroissance de l'erreur avec $\epsilon_{D F}$ dûe au gain de précision de l'approximation par différences finies (développement de Taylor). Puis, on constate une remontée de l'erreur, signe que la précision machine est atteinte (de l'ordre de $10^{-14}$ car nous travaillons en "double précision").

La figure 3 présente les pentes correspondantes (ordre 1 à gauche et ordre 2 à droite). Conformément à la théorie, on retrouve bien une pente de 1 pour la différentielle décentrée d'ordre 1 et une pente de 2 pour les différentielles centrées d'ordre 1 et 2 .

Enfin, le dernier temps (étape 3) correspond à la validation globale des deux types de sensibilité seconde (paramètres $\eta$ et $\nu$ ). Voici les paramètres retenus: 

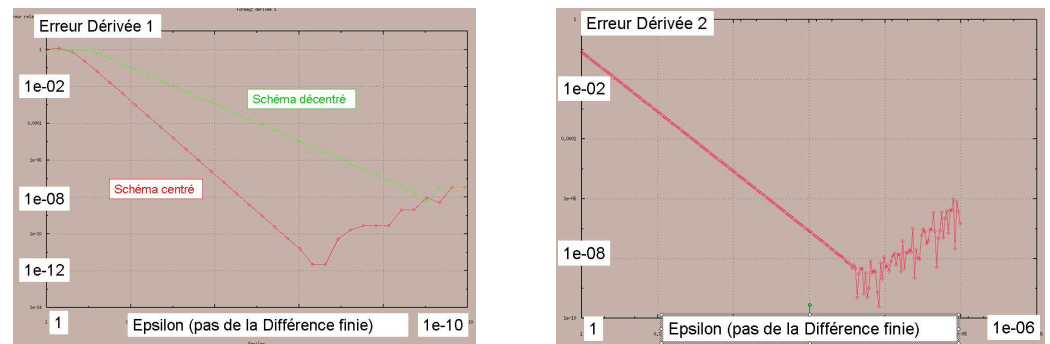

Figure 2. Courbe de l'erreur absolue issue de la comparaison avec la différence finie
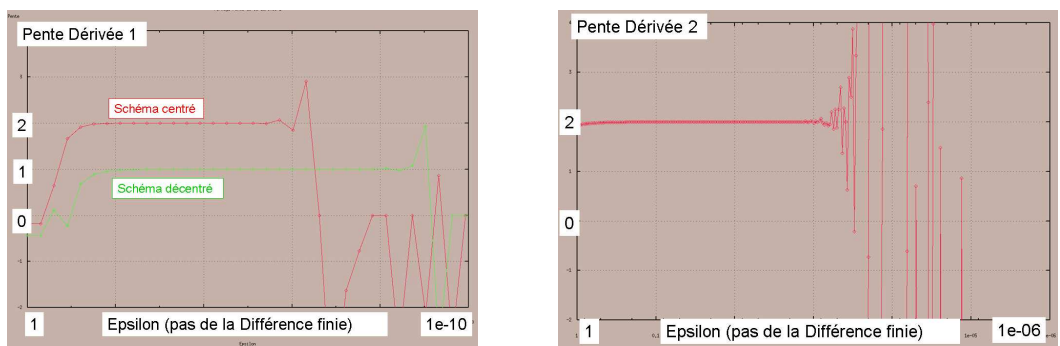

Figure 3. Pente issue de la comparaison avec la différence finie

- Pour la classe $\eta$ (pas d'influence sur le maillage), on prend les angles d'incidence $\alpha_{\eta}$ et de dérapage $\beta_{\eta}$. Notons que ces angles pilotent la direction de l'écoulement à l'infini.

- Pour la classe $\nu$ (influence sur le maillage), on prend deux angles de rotation $\left(\alpha_{\nu}, \beta_{\nu}\right)$ pour le maillage global. Ces derniers produisent le même effet relatif que les angles d'incidence et de dérapage $\left(\alpha_{\eta}, \beta_{\eta}\right)$, mais par rotation de l'objet cette fois.

Il faut remarquer que ces paramètres $\left(\alpha_{\nu}, \beta_{\nu}\right)$ de rotation donnent directement le champ de déplacement volumique sans passer par un déplacement surfacique défini uniquement sur l'objet. Cela correspond au cas 1 décrit lors de la première section de cet article.

Dans toute la suite, comme les deux jeux de paramètres ont le même effet relatif, l'incidence (resp. le dérapage) désignera indistinctement $\alpha_{\eta}$ ou $\alpha_{\nu}$ (resp. $\beta_{\eta}$ ou $\left.\beta_{\nu}\right)$ ). En revanche, numériquement, ces deux jeux de paramètres ne donneront pas rigoureusement le même résultat, dû au fait que le maillage n'est pas infiniment fin.

On s'attend cependant à trouver un résultat de sensibilité très proche pour les deux classes de paramètres. Les résultats numériques concernent deux cas-test (fuselage en supersonique et l'aile ONERA M6 en transsonique) et sont exposés dans la section suivante.

\section{RÉSultats NUmÉRiQues DE VALIDATION}

\subsection{Cas-test du fuselage en supersonique}

Le premier cas-test concerne un fuselage simple représenté par la figure 4. La longueur totale est de 40 mètres et son diamètre maximal de 2 mètres environ. On fixe le nombre de Mach à 1,6. On est donc en présence d'un écoulement supersonique. Le maillage, découpé en 32 sous-domaines, comporte 152825 sommets. Notre objectif est de calculer les sensibilités du coefficient de traînée $C x$ vis-à-vis des angles $\left(\alpha_{\eta}, \beta_{\eta}\right)$ de classe $\eta$ (car n'influant pas le maillage) et des angles $\left(\alpha_{\nu}, \beta_{\nu}\right)$ de classe $\nu$ (car influant le maillage). La figure 5 montre la surface obtenue grâce à un ensemble de simulations. Sur la plage de variation des angles d'incidence et de dérapage considérés (de 0 à 8 degrés pour les deux), la traînée est comprise entre 45 et 75 points. Notons que 

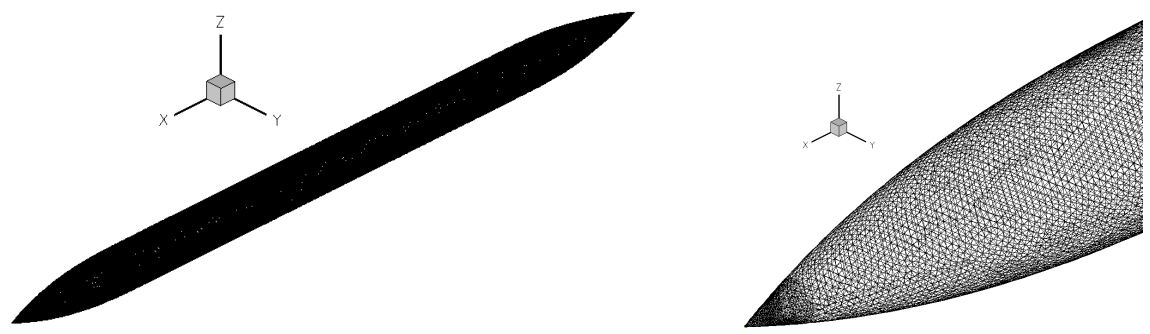

Figure 4. Représentation du fuselage

la différence de résultat entre les deux classes de paramètres n'excède pas $10^{-7}$ point de traînée ${ }^{2}$. De plus, dans toute la suite, les paramètres de type $\nu$ envisagés appartiennent à la seconde classe, pour laquelle le champ de déplacement volumique $D$ est obtenu directement (sans l'intervention du déplacement surfacique $d$ ).

Le premier moyen de validation est de représenter géométriquement les sensibilités première et seconde en
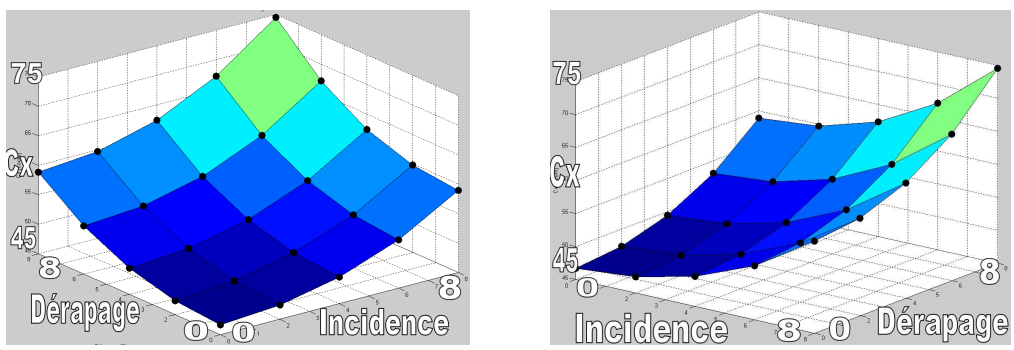

Figure 5. Surface de la traînée Cx pour différentes valeurs d'angles d'incidence et de dérapage (fuselage)

quelques points, deux ici (figure 6). On voit en vert les tangentes et en rouge une partie des cercles osculateurs dans les deux directions principales. On a superposé les résultats issus des deux classes de paramètres (sphères pour la classe $\eta$ et points pour la classe $\nu$ ). A la vue de ces figures, on conclue à:
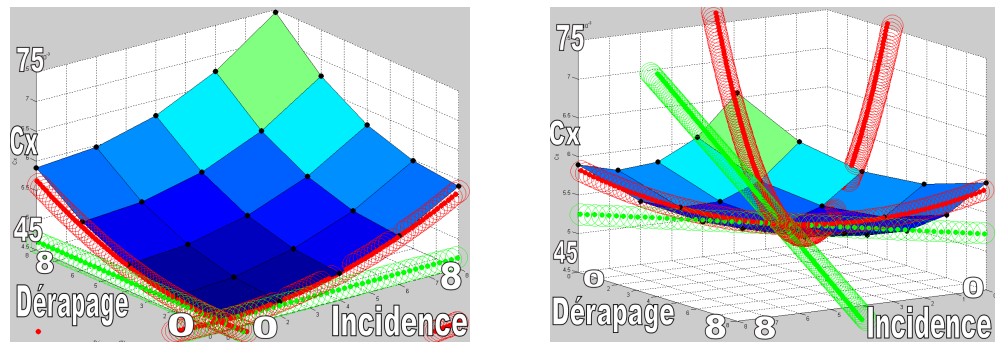

Figure 6. Tangentes et courbures géométriques obtenues (fuselage)

- la très bonne similarité des sensibilités entre les deux types de paramètres

- la bonne adéquation des sensibilités avec l'allure générale de la surface

Afin de compléter l'étude, on peut analyser la qualité de prédiction via un développement de Taylor utilisant les sensibilités calculées. La figure 7 situe les points utilisés par la prédiction dans les deux configurations envisagées (1 point pour la première configuration et 4 pour la seconde). Pour reconstruire la surface à partir

\footnotetext{
${ }^{2}$ l'unité utilisée est le point de traînée, qui revient à un coefficient multiplicatif de $10^{-4}$
} 

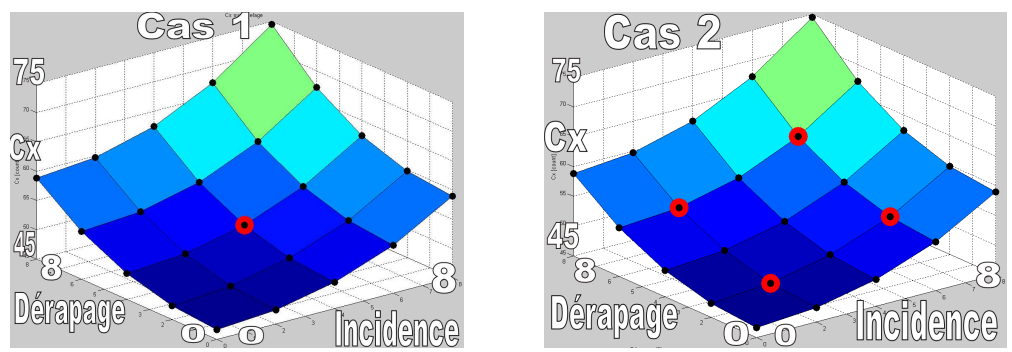

Figure 7. Positions des points de reconstruction dans les deux configurations envisagées (fuselage)

de ces points sélectionnés, on procède comme suit. La valeur de la traînée en un point quelconque sera égale au développement de Taylor du point sélectionné le plus proche dans l'espace des paramètres. La figure 8 représente, pour la configuration à un point, les surfaces prédites à l'ordre 1 (figure de gauche, avec des paramètres $\left(\alpha_{\eta}, \beta_{\eta}\right)$ appartenant à la classe $\eta$ ) et à l'ordre 2 (figures de droite, avec ces mêmes paramètres $\left(\alpha_{\eta}, \beta_{\eta}\right)$ de type $\eta$ pour la figure centrale et des paramètres $\left(\alpha_{\nu}, \beta_{\nu}\right)$ appartenant à la classe $\nu$ pour la figure de droite). La figure 9 présente la même chose mais pour la configuration à quatre points.
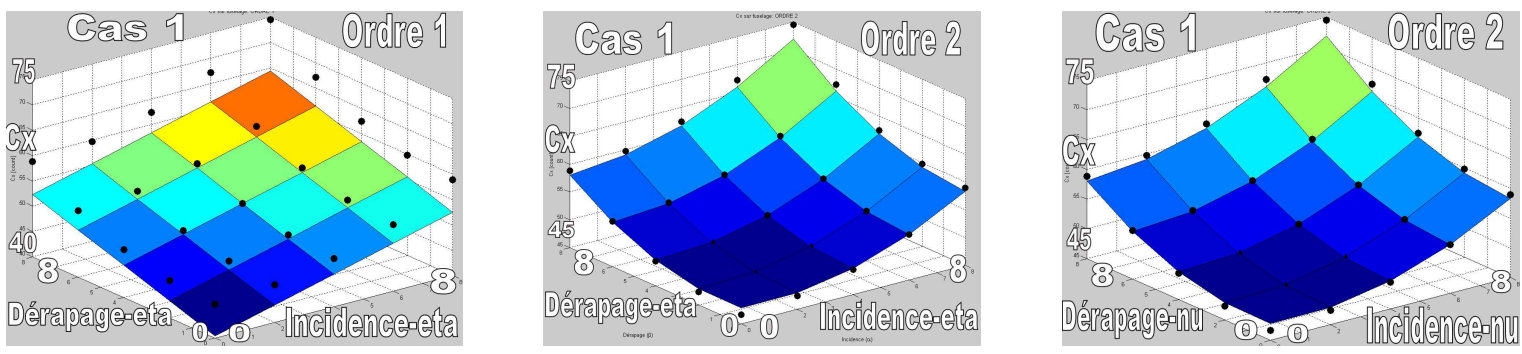

FiguRE 8. Forme des surfaces prédites pour la première configuration (fuselage)
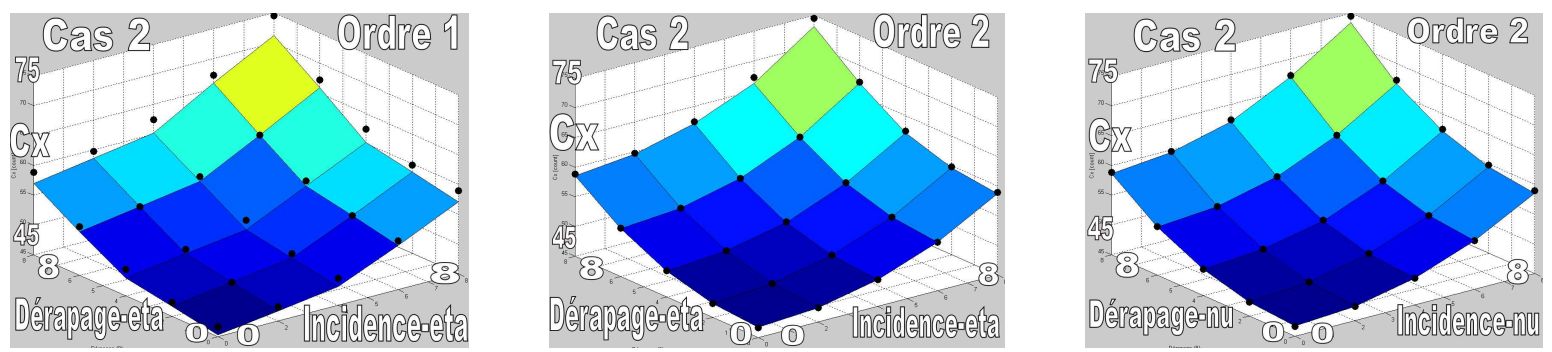

Figure 9. Forme des surfaces prédites pour la seconde configuration (fuselage)

On note les ruptures de pente dans le cas de l'ordre 1 qui n'apparaîssent plus à l'ordre 2. Afin de mesurer plus précisément l'amélioration dûe à la montée en ordre, les figures 10 et 11 affichent les différences absolues entre les surfaces réelle et prédite pour les configurations considérées. Bien sûr, pour une même configuration, on a gardé une échelle identique.

On peut compléter ces informations par les valeurs moyennes numériques (en points de traînée) de cette erreur de prédiction:

- Pour la configuration à 1 point, à l'ordre 1: 3,59 (classe $\eta$ ) et 3,59 (classe $\nu$ )

- Pour la configuration à 1 point, à l'ordre 2: $5,06 \cdot 10^{-1}$ (classe $\eta$ ) et $5,07.10^{-1}$ (classe $\nu$ ) 

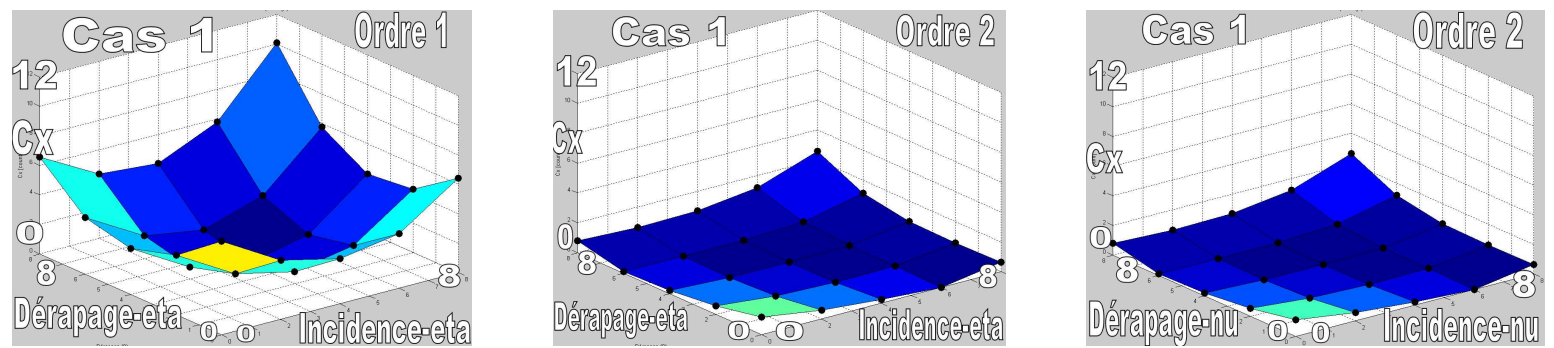

FiguRE 10. Différence absolue entre les surfaces prédites et réelles pour la première configuration (fuselage)
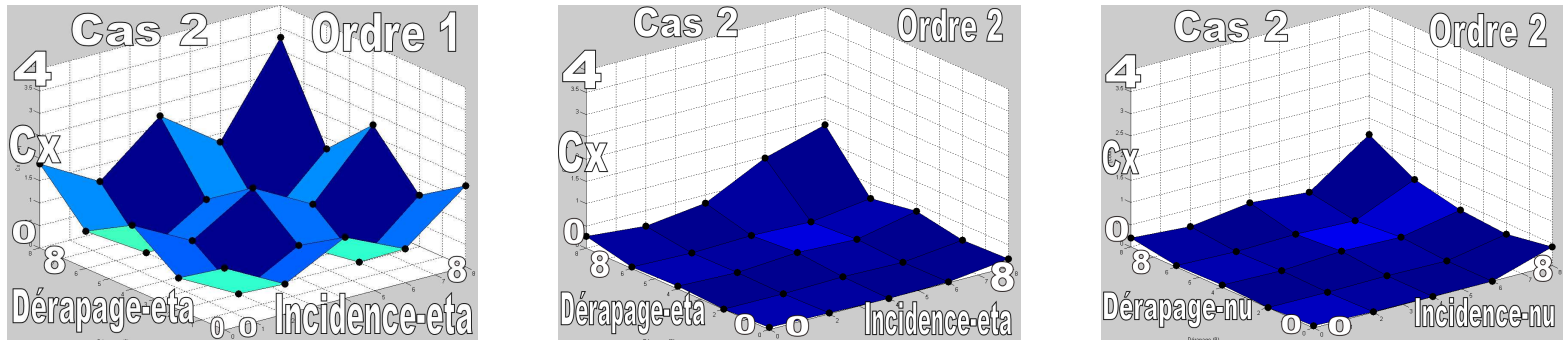

FiguRE 11. Différence absolue entre les surfaces prédites et réelles pour la seconde configuration (fuselage)

- Pour la configuration à 4 points, à l'ordre 1: 1,12 (classe $\eta)$ et $1,12($ classe $\nu)$

- Pour la configuration à 4 points, à l'ordre $2: 1,75.10^{-1}$ (classe $\eta$ ) et $1,57.10^{-1}$ (classe $\nu$ )

De ces informations, il apparaît que:

- Comme attendu, l'erreur de prédiction est d'autant plus importante que le point est loin de ceux sélectionnés préalablement pour mener la reconstruction

- Les gains réalisés par l'utilisation des dérivées secondes sont significatifs. Ainsi, si on divise par 3 l'erreur moyenne de prédiction en augmentant le nombre de points sélectionnés pour la reconstruction de la surface (passage de la configuration à 1 point à la configuration à 4 points), un facteur égal à 7 environ apparaît lors de la montée en ordre pour chacune des deux configurations.

Cet exemple permet de mieux comprendre le rôle fondamental de l'ordre 2 pour diminuer autant que possible le nombre de simulations sans perdre en précision.

On note que ce cas-test a été choisi car il offre une surface présentant une symétrie, dûe à la symétrie remarquable de l'objet considéré (et également à la symétrie vis-à-vis des angles d'incidence et de dérapage). Nous allons maintenant considérer un cas-test qui ne comporte plus ce type de symétrie géométrique (axisymétrie du fuselage), ni de symétrie des effets incidence et dérapage.

\subsection{Cas-test de l'aile ONERA M6 en transsonique}

La seconde étude menée pour valider le calcul de la sensibilité seconde des observations aérodynamiques concerne l'aile ONERA M6. Le nombre de Mach est fixé à 0,84 (écoulement transsonique). Ce cas-test de référence est illustré par la figure 12. Celle de gauche dévoile le maillage surfacique avec une couleur différente par domaine tandis que celle de droite montre la répartition de la pression locale pour une incidence de 4 degrés et un dérapage nul. On retrouve bien le choc en "lambda" typique de cette simulation.

Le maillage choisi comporte 52886 sommets et est découpé en 8 sous-domaines. On reprend les mêmes paramètres (incidence et dérapage) et la même fonction d'intérêt (coefficient de traînée $C x$ ) que pour le cas-test du fuselage. La surface de réponse est construite à nouveau via un ensemble de simulations (figure 13). On 

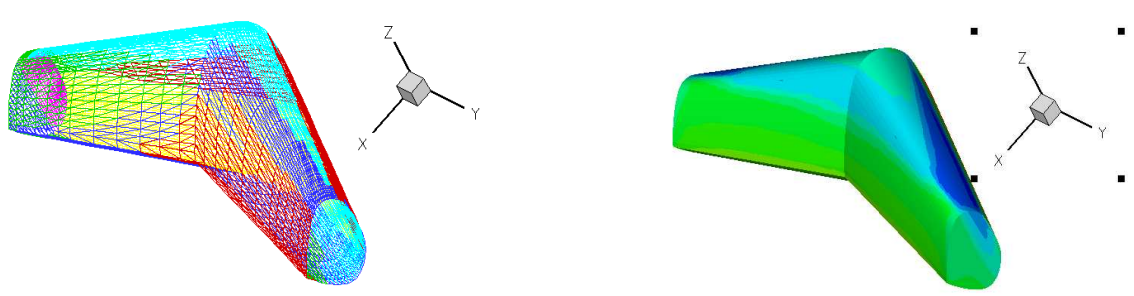

FiguRE 12. Maillage et distribution de pression locale pour l'aile ONERA M6

considère ici des angles d'incidence et de dérapage bien plus grands que dans les études habituelles pour l'aile ONERA M6, mais ceci nous permet d'obtenir une topologie suffisamment riche pour y visualiser les sensibilités. Sur la plage de variation des angles d'incidence et de dérapage considérés (de 4 à 10 degrés pour l'incidence et de 0 à 30 degrés pour le dérapage), la traînée est comprise entre 150 et 1700 points. Nous appuyons sur le fait que, les angles d'incidence et de dérapage pouvant prendre des valeurs bien supérieures à celles considérées dans la pratique, la traînée obtenue peut atteindre des valeurs considérables. Néanmoins, cette exploration était utile à la démonstration numérique recherchée (intérêt des dérivées secondes). Il ne faut pas s'attacher à la validité physique des valeurs de traînée obtenues pour ces grands angles d'incidence et de dérapage.

La première constatation est que cette surface ne présente pas l'aspect symétrique du premier cas-test. Comme
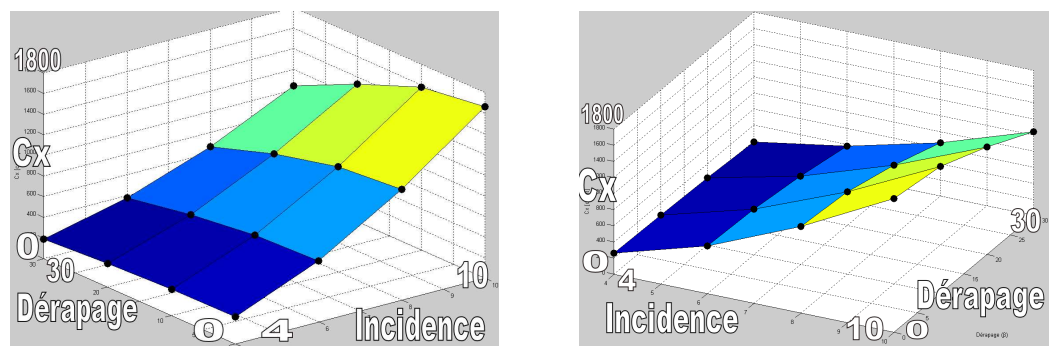

Figure 13. Surface de la traînée Cx pour différentes valeurs d'angles d'incidence et de dérapage (aile ONERA M6)

pour le précédent cas-test, la figure 14 représente géométriquement les sensibilités première et seconde en quelques points dans les directions principales. Les tangentes sont en vert alors que les morceaux de cercles osculateurs sont en rouge. On a superposé les résultats issus des deux classes de paramètres (sphères pour la classe $\eta$ et points pour la classe $\nu$ ). A nouveau, la correspondance entre les deux manières de calculer les
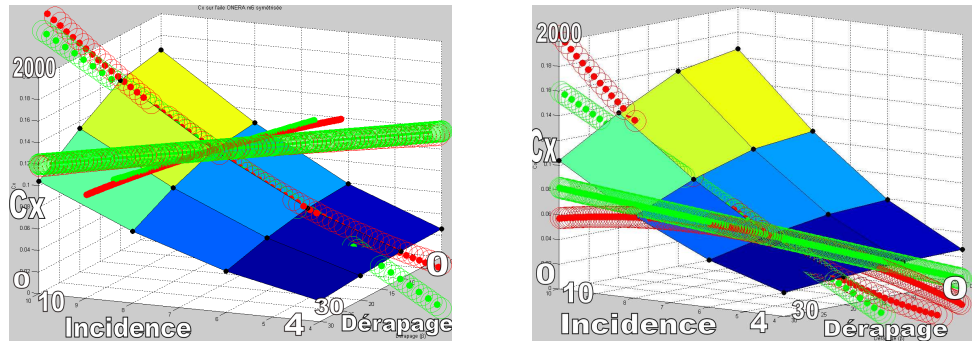

Figure 14. Tangentes et courbures géométriques obtenues (aile ONERA M6)

dérivées est tout à fait acceptable (on note cependant un léger décalage pour la direction suivant laquelle il 
s'avère que la fonction ne varie presque pas, ce qui dénote un simple problème de précision). De plus, les cercles osculateurs permettent de prédire presque parfaitement les points voisins.

Nous allons maintenant construire une approximation de la surface à partir de deux points, dont les emplacements dans deux configurations sont donnés en figure 15.
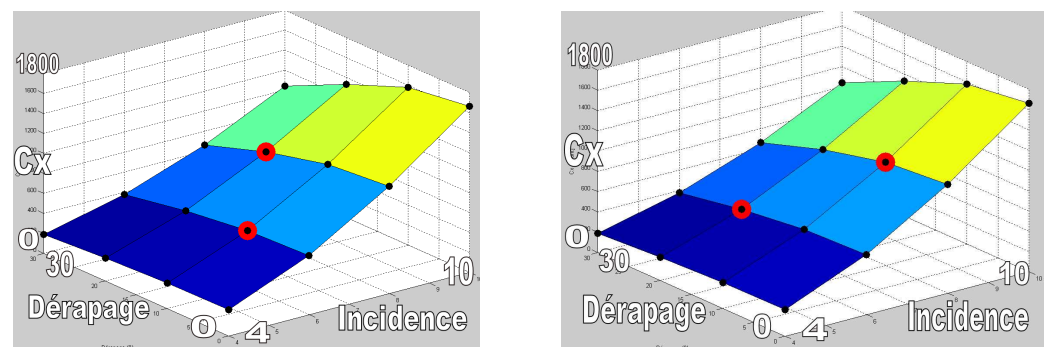

Figure 15. Positions des points de reconstruction dans les deux configurations envisagées (aile ONERA M6)

L'erreur absolue entre les surfaces exacte et prédite (figures 16 et 17) montrent à nouveau que l'erreur est importante loin des points sélectionnés (dans ce cas, au niveau des coins). Le passage à l'ordre 2 améliore nettement les résultats.
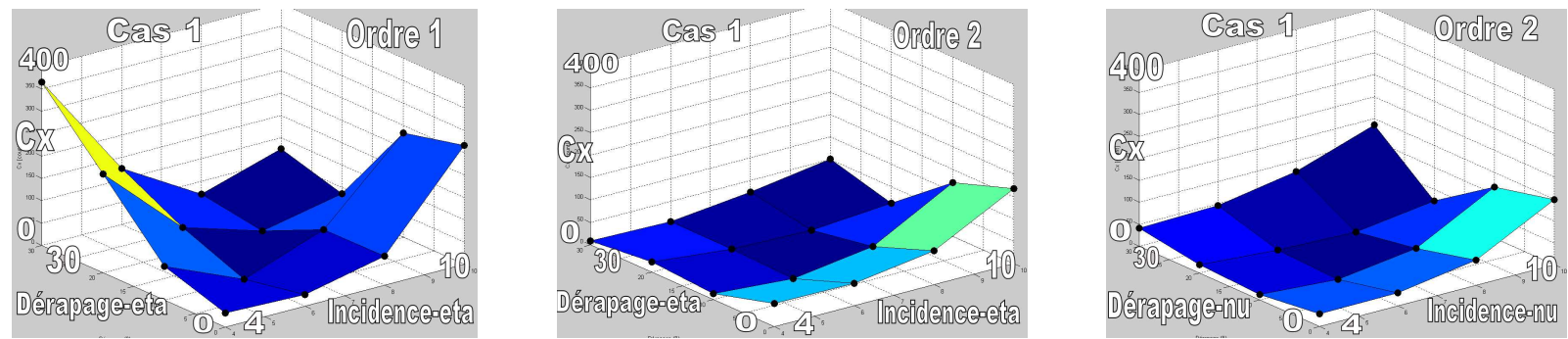

Figure 16. Différence absolue entre les surfaces prédites et réelles pour la première configuration (aile ONERA M6)
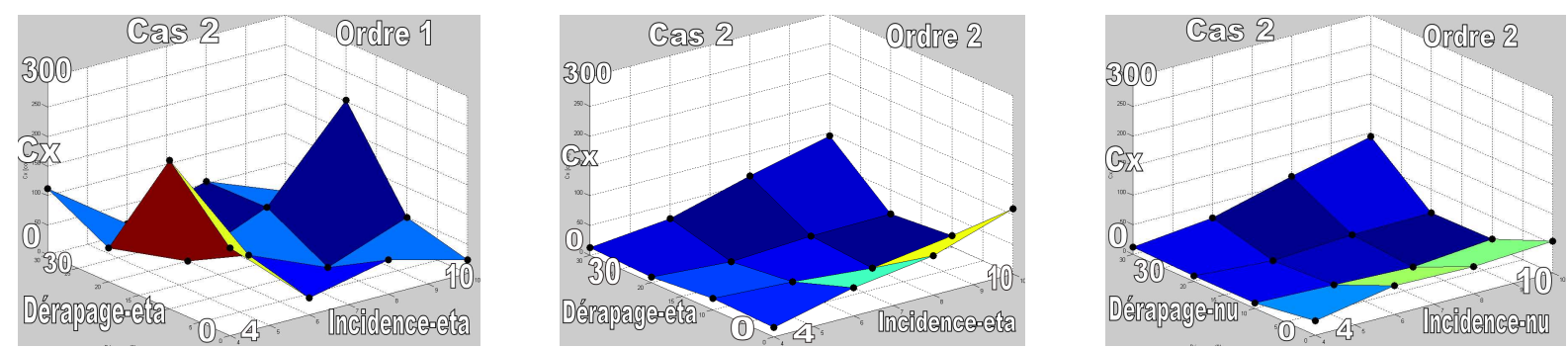

FiguRE 17. Différence absolue entre les surfaces prédites et réelles pour la seconde configuration (aile ONERA M6)

Ces constatations sont confortées par les valeurs moyennes numériques (en points de traînée) de l'erreur de prédiction:

- Pour la configuration 1, à l'ordre 1: 103, 74 (classe $\eta$ ) et 103, 73 (classe $\nu$ )

- Pour la configuration 1, à l'ordre 2: 39, 46 (classe $\eta$ ) et 47,78 (classe $\nu$ ) 
- Pour la configuration 2, à l'ordre 1: 69,77 (classe $\eta$ ) et 69,80 (classe $\nu$ )

- Pour la configuration 2, à l'ordre 2: 32,13 (classe $\eta$ ) et 26,31 (classe $\nu$ )

Ici, la montée en ordre permet de diviser l'erreur moyenne par un facteur compris entre 2 et 3 . Compte tenu de la forme de la surface à prédire, ceci nous conforte à la fois sur la validité des sensibilités implémentées et dans le choix de passer à l'ordre 2 .

\section{Propagation des incertitudes}

Nous détaillons dans cette section un autre exemple d'utilisation pratique des sensibilités, permettant cette fois de mettre en lumière l'importance de l'ordre 2 pour la propagation des incertitudes [7]. L'objectif général de cette section est de connaître l'impact de l'incertitude concernant les paramètres d'entrée sur notre fonction de sortie (ou réponse). En particulier, on est intéressé par la valeur de sa moyenne et de son écart-type. Pour cela, deux méthodes sont ici utilisées, à savoir la méthode des moments et la méthode Monte-Carlo sur le développement de Taylor.

\subsection{Présentation des méthodes retenues}

La méthode des moments n'est rien d'autre que l'expression analytique des premiers moments de la réponse connaissant le type de loi que suivent les variables d'entrée. Ainsi, notons $\left(v_{i}\right)_{1 \leq i \leq N_{e}}$ les $N_{e}$ variables d'entrée et $f$ notre réponse. On suppose que ces variables d'entrée $v_{i}$ subissent chacune une perturbation $\Delta_{i}$ sous forme d'une variable aléatoire centrée. On suppose également que les perturbations $\Delta_{i}$ sont deux à deux indépendantes. On désigne par $E_{i}$ (resp. par $\sigma_{i}$ ) la moyenne (resp. l'écart-type) associée à $\Delta_{i}$. On rappelle que, par hypothèse, les moyennes $E_{i}$ sont toutes nulles.

Dans ces conditions, la moyenne $E_{f}$ de notre réponse est:

$$
\left\{\begin{array}{l}
E_{f}=f\left(v_{1}, \ldots, v_{N_{e}}\right)(\text { ordre } 1) \\
E_{f}=f\left(v_{1}, \ldots, v_{N_{e}}\right)+\frac{1}{2} \sum_{i=1}^{N_{e}} \frac{\partial^{2} f}{\partial v_{i}^{2}} \sigma_{i}^{2}(\text { ordre } 2)
\end{array}\right.
$$

On s'aperçoit en particulier que l'ordre 1 n'apporte aucune information sur la moyenne, contrairement à l'ordre 2. La variance $\sigma_{f}^{2}$ de notre réponse, quant à elle, vaut:

$$
\left\{\begin{array}{l}
\sigma_{f}^{2}=\sum_{i=1}^{N_{e}}\left(\frac{\partial f}{\partial v_{i}}\right)^{2} \sigma_{i}^{2}(\text { ordre 1) } \\
\sigma_{f}^{2}=\sum_{i=1}^{N_{e}}\left(\frac{\partial f}{\partial v_{i}}\right)^{2} \sigma_{i}^{2}+\frac{1}{2} \sum_{i=1}^{N_{e}}\left(\frac{\partial^{2} f}{\partial v_{i}^{2}}\right)^{2}\left(E\left(\Delta_{i}^{4}\right)-\sigma_{i}^{4}\right)+\sum_{i=1}^{N_{e}} \sum_{j=(i+1)}^{N_{e}}\left(\frac{\partial^{2} f}{\partial v_{i} \partial v_{j}}\right)^{2} \sigma_{i}^{2} \sigma_{j}^{2} \text { (ordre 2) }
\end{array}\right.
$$

où $E\left(\Delta_{i}^{4}\right)$ désigne l'espérance de la variable aléatoire $\Delta_{i}^{4}$.

La seconde méthode choisie est la méthode Monte-Carlo sur le développement de Taylor. La première étape consiste à écrire les développements de Taylor de notre réponse $f$ à l'ordre 1 et 2.

$$
\left\{\begin{aligned}
f\left(v_{1}+\Delta_{1}, \ldots, v_{N_{e}}+\Delta_{N_{e}}\right) & =f\left(v_{1}, \ldots, v_{N_{e}}\right)+\sum_{i=1}^{N_{e}} \frac{\partial f}{\partial v_{i}} \Delta_{i} \text { (ordre 1) } \\
f\left(v_{1}+\Delta_{1}, \ldots, v_{N_{e}}+\Delta_{N_{e}}\right) & =f\left(v_{1}, \ldots, v_{N_{e}}\right)+\sum_{i=1}^{N_{e}} \frac{\partial f}{\partial v_{i}} \Delta_{i}+\frac{1}{2} \sum_{i=1}^{N_{e}} \sum_{j=1}^{N_{e}} \frac{\partial^{2} f}{\partial v_{i} \partial v_{j}} \Delta_{i} \Delta_{j} \text { (ordre 2) }
\end{aligned}\right.
$$

Ensuite, on effectue un ensemble de tirages sur les paramètres d'entrée $v_{i}+\Delta_{i}$ en respectant les lois que suivent chacune de ces grandeurs. Pour chacun de ces tirages, on peut calculer la valeur de notre réponse via l'approximation donnée par les développements de Taylor ci-dessus. Il ne reste ensuite plus qu'à estimer la moyenne et l'écart-type à partir de l'ensemble de valeurs de notre réponse $f$ ainsi constitué. On note que, par rapport à la méthode des moments, on rajoute une approximation supplémentaire lors de l'évaluation de la moyenne et de l'écart-type de notre réponse, qui est proportionnelle au nombre de tirages effectués. Cependant, l'avantage d'utiliser une méthode de type Monte-Carlo approché est de pouvoir connaître la forme de la loi de probabilité en sortie. 


\subsection{Résultats obtenus}

On reprend le cas-test précédent de l'aile ONERA M6 pour un écoulement transsonique (Mach égal à 0,84). Le but est de propager une incertitude sur les angles d'incidence et de dérapage pour voir l'impact sur la valeur de la traînée.

Plus précisement, on suppose que chacun de ces deux angles suit une loi normale centrée (ie de moyenne nulle) et d'écart-type égal à 1 point de traînée. Une autre hypothèse importante est que ces deux variables aléatoires sont indépendantes. On veut connaître le comportement de la traînée qui devient par conséquent elle aussi une variable aléatoire. En particulier, on est intéressé par la valeur de sa moyenne et de son écart-type.

On déroule les deux méthodes de propagation d'incertitude décrite précédemment autour des deux points de référence suivants:

- Point $A$ : incidence égale à 4 et dérapage égal à 10 , la traînée valant 252,61 (en points)

- Point $B$ : incidence égale à 8 et dérapage égal à 0 , la traînée valant 1073, 3 (en points)

Notons que la méthode Monte-Carlo est effectuée avec 10000 tirages. Pour avoir une idée de la préçision, notons que la moyenne (resp. l'écart-type) des tirages de l'incidence donne $1,06.10^{-3}$, à comparer avec 0 (resp. 1.001 , à comparer avec 1$)$.

Les tableaux 1 (resp. 2) récapitulent les résultats obtenus concernant la moyenne (resp. l'écart-type) de la traînée. Remarquons qu'on a introduit l'incertitude uniquement au niveau de l'incidence, puis uniquement au niveau du dérapage, pour terminer par le cas où l'incertitude touche les deux angles.

\begin{tabular}{|c|c|c|c||c|c||c|c|}
\hline \multicolumn{2}{|c|}{ Variables aléatoires } & \multicolumn{2}{|c||}{ Incidence } & \multicolumn{2}{c||}{ Dérapage } & \multicolumn{2}{c|}{ Incidence-Dérapage } \\
\hline \hline Point & méthode & Ordre 1 & Ordre 2 & Ordre 1 & Ordre 2 & Ordre 1 & Ordre 2 \\
\hline Pt A & Moments & 252,61 & 272,77 & 252,61 & 252,5 & 252,61 & 272,66 \\
\hline Pt A & Monte-Carlo & 252,74 & 272,94 & 252,6 & 252,49 & 252,73 & 272,81 \\
\hline \hline Pt B & Moments & 1073,3 & 1089,8 & 1073,3 & 1073,3 & 1073,3 & 1089,7 \\
\hline Pt B & Monte-Carlo & 1073,6 & 1090,1 & 1073,3 & 1073,3 & 1073,6 & 1090 \\
\hline
\end{tabular}

TABle 1. Moyennes sur la traînée Cx (en points)

\begin{tabular}{|c|c|c|c||c|c||c|c|}
\hline \multicolumn{2}{|c|}{ Variables aléatoires } & \multicolumn{2}{|c||}{ Incidence } & \multicolumn{2}{c||}{ Dérapage } & \multicolumn{2}{c|}{ Incidence-Dérapage } \\
\hline \hline Point & méthode & Ordre 1 & Ordre 2 & Ordre 1 & Ordre 2 & Ordre 1 & Ordre 2 \\
\hline Pt A & Moments & 118,24 & 121,63 & 1,7699 & 1,7772 & 118,25 & 121,65 \\
\hline Pt A & Monte-Carlo & 118,37 & 122 & 1,7762 & 1,7843 & 118,36 & 121,99 \\
\hline \hline Pt B & Moments & 270,66 & 271,65 & $6.0072 .10^{-10}$ & $7.4336 .10^{-2}$ & 270,66 & 271,65 \\
\hline Pt B & Monte-Carlo & 270,95 & 272,08 & $6.0287 .10^{-10}$ & $7.607 .10^{-2}$ & 270,95 & 272,08 \\
\hline
\end{tabular}

TABLE 2. Ecart-types sur la traînée Cx (en points) 
A la vue de ces tableaux, on peut dire que:

- comme on a pu s'en rendre compte, la traînée est beaucoup moins sensible au dérapage qu'à l'incidence. Cet aspect se traduit par la quasi-constance de la moyenne lorsque seul le dérapage est incertain et la petitesse relative de l'écart-type correspondant. Il se remarque aussi en se rendant compte de la similitude des résutats obtenus entre les cas où l'incidence est la seule source d'incertitude et où les deux angles sont incertains. Dans la suite, on ne distinguera donc plus ces deux cas; on ne considèrera qu'une incertitude sur l'angle d'incidence.

- la différence entre les deux méthodes restent relativement faibles et du même ordre dans tous les cas (inférieur à 1 point de traînée). Cela n'a rien d'étonnant dans la mesure où cette différence est inversement proportionnelle au nombre de tirages utilisé par la méthode Monte-Carlo.

- concernant le passage de l'ordre 1 à l'ordre 2 dans le cas du dérapage incertain, c'est autour du point $B$ que la différence d'écart-type est la plus spectaculaire (la moyenne restant quant à elle quasiment constante). En effet, le dérapage étant nul et l'aile étant symétrique vis-à-vis de cet angle, le dérivée première de la traînée vis-à-vis du dérapage est nul en ce point. L'ordre 1 ne donne ainsi aucune information (écart-type négligeable), contrairement à l'ordre 2. Pour ce qui est de l'incidence, la montée en ordre provoque dans tous les cas un écart similaire sur la moyenne (20 points environ). Concernant l'écart-type en incidence, c'est le point $A$ qui présente une différence un peu plus importante (3 à 4 contre 1 à 2 points).

Pour illustrer la différence venant de la dernière remarque formulée, on peut s'aider des histogrammes des variables aléatoires issus de la méthode Monte-Carlo. La figure 18 montre les histogrammes de l'incidence et du dérapage (loi normale centrée d'écart-type 1), tandis que la figure 19 (resp. 20) montre les histogrammes de la traînée à l'ordre 1 et 2 pour le point $A$ (resp. pour le point $B$ ).
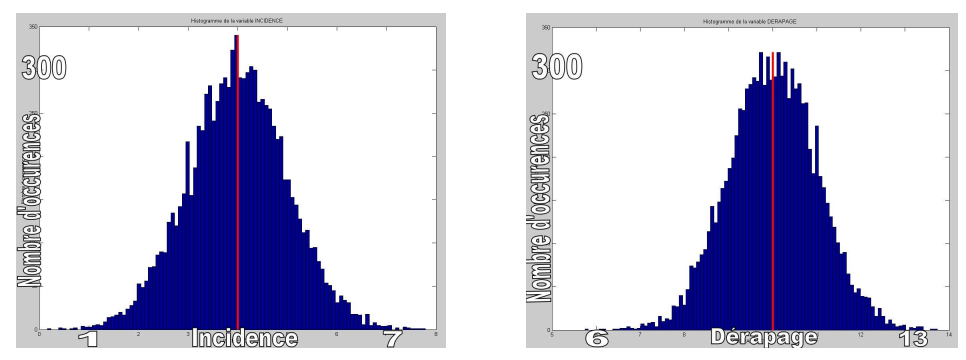

Figure 18. Histogramme de l'incidence (à gauche) et du dérapage (à droite)
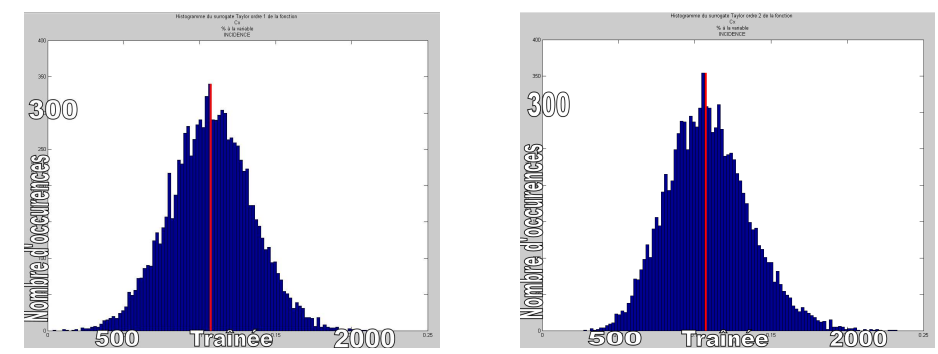

Figure 19. Histogramme de la traînée pour le point $A$ à l'ordre 1 (à gauche) et 2 (à droite) avec une incertitude sur l'incidence

L'aspect à retenir de ces figures est que, pour le point $B$, on retrouve une loi centrée pour la traînée (ordre 1 et 2), similaire à la forme des lois en entrée, contrairement au cas du point $A$, pour lequel apparaît une loi 

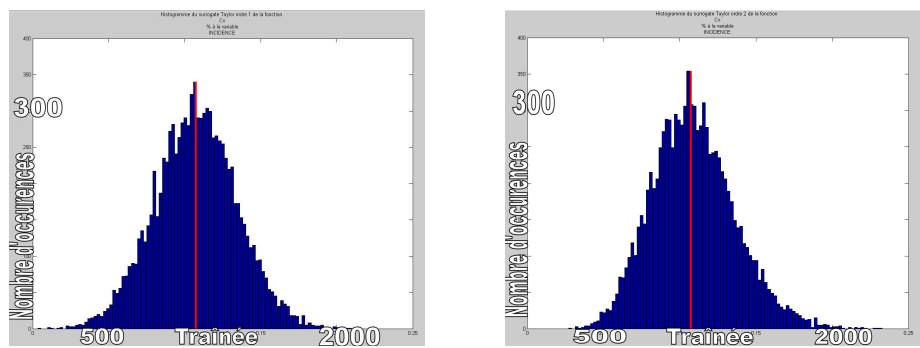

Figure 20. Histogramme de la traînée pour le point $B$ à l'ordre 1 (à gauche) et 2 (à droite) avec une incertitude sur l'incidence

normale tronquée (uniquement à l'ordre deux), dû à la présence d'un minimum $\left(\frac{\partial C_{x}}{\partial \beta}=0\right)$.

$\mathrm{Au}$ cours de cet article, l'importance de la prise en compte des sensibilités d'ordre deux a pu être mise en lumière, ainsi que la manière de la mettre en place puis de la valider au sein d'un code de calcul industriel. Moyennant un effort à fournir pour son implémentation, cette donnée n'est pas seulement un luxe de précision mais s'avère souvent porteuse d'une information très riche, et ceci à un coût de calcul admissible.

Un grand merci à S. Kleinveld pour la relecture de cet article et à L. Daumas pour son aide dans la compréhension du fonctionnement du code de calcul industriel utilisé.

This work was supported by the project NODESIM-CFD "Non-deterministic Simulation for CFD-based Design Methodologies" funded by the European Comunity represented by the CEC, Research Directorate-General, in the 6th Framework Programme, under Contract No. AST5-CT-2006-030959.

\section{REFERENCES}

[1] D. P. Ghate et M. B. Giles, Efficient Hessian Calculation using Automatic Differentiation 25th AIAA Applied Aerodynamics Conference, 25-28 June 2007, Miami, FL

[2] L. Hascoët, A. Dervieux et V. Pascual, Software TAPENADE INRIA 2002, versions 2.0 and later hereinafter "the software" , developpés par des chercheurs du projet TROPICS à l'INRIA (Institut National de Recherche en Informatique et Automatique)

[3] Q. Dinh, G. Rogé, C. Sevin et B. Stoufflet, Shape Optimisation in Computational Fluid Dynamics, European Journal of Finite Elements, Vol. 5, p. 569-594, 1996

[4] F. Alauzet, S. Borel-Sandou, L. Daumas, A. Dervieux, Q. Dinh, S. Kleinveld, A. Loseille, Y. Mesri et G. Rogé, Multimodel design strategies applied to sonic boom reduction, REMN - 17/2008. Shape design in aerodynamics, pages 245 to 269

[5] J. Kybic, T. Blu et M. Unser, Generalized Sampling: A Variational Approach - Part I: Theory, IEEE TRANSACTIONS ON SIGNAL PROCESSING, vol. 50, no. 8, August 2002

[6] J. Kybic, T. Blu et M. Unser, Generalized Sampling: A Variational Approach - Part II: Applications, IEEE TRANSACTIONS ON SIGNAL PROCESSING, vol. 50, no. 8, August 2002

[7] D. Pelletier, Uncertainty Analysis by the Sensitivity Equation Method, 41st AIAA Aerospace Sciences Meeting and Exhibit, 6-9 January 2003

[8] J. Bastin et G. Rogé, A Multidimensional Fluctuation Splitting Scheme for the three Dimensional Euler Equations, Mathematical Modelling and Numerical Analysis, vol. 33, no. 6, 1999, p. 1241-1259 